\title{
Forum
}

\section{Review of Mathematical Methods}

\author{
Roy Williams
}

Reading through the paper by Carlton Wippern in the May 1992 edition of the Journal makes me realise that the time has definitely arrived when we should analyse all of our methods of computation in navigation using modern methods of mathematical analysis.

The traditional educational pattern in navigation, particularly for seagoing navigators, seems to skilfully avoid the use of calculus, yet, as long ago as $192 \mathrm{I}$, in his book The Mathematics of Navigation, the American author Edward J. Willis recommended the use of the calculus as a method of deriving the familiar navigational formulae which is easier than spherical trigonometry, and this is a view I endorse.

In this modern age we must look forward to the technicalities of navigating on the surfaces of other planets. Some of these planets, such as Venus, Mars and Mercury, are very similar in shape to Earth and our 'Earthbound' methods may well serve as good approximations on those surfaces too but, supposing that it is technically possible and that we were to consider sending a space vehicle to skim the surface of the outer planets such as Jupiter and Saturn, where the flattening of the surface is very distinct, then the methods of spherical trigonometry will not apply and we will need the best mathematical analysis to analyse curves on such a surface. This analysis can only be achieved using the methods of differential geometry. The shortest path geodesic arcs on the surface of a distinct spheroid are sinusoidal curves with greatly varying sinusoidal periods (unlike the great circles on the surface of a sphere which all have a fixed period of $360^{\circ}$ ), and I have not seen a method of computation that will satisfactorily solve the problem of determining the shortest distance between two points along a geodesic arc in these circumstances. The only method that I have seen that would satisfactorily solve the problem of navigating along the path of a loxodromic curve on the surface of a distinct spheroid, and which does not employ the use of tables, is the method by Carlton Wippern. His method can be applied equally well on the surface of a sphere or spheroid.

\section{'Air Navigation Systems'}

\section{from Alan Sillitoe}

In the essay 'Air Navigation Systems' (vol. 44, September 1991) it is written: 'Very little has been made public about radio navaid developments in the USSR'.

For several years I have been intercepting $w / T$ signals from Soviet civilian and military aircraft overflying European Russia and Siberia in contact with a high frequency direction finding (HF/DF) station transmitting on $11380 \mathrm{kHz}$. Very few aircraft use this service, and sometimes there are only half a dozen calls a day. 
The aircraft calls the station (UAZL) and requests a QTF (position in latitude and longitude). UAZL asks for a QTG, which as I am sure readers will know means that the operator in the aircraft presses his morse key for 10 seconds or so. Then, after about a minute, UAZL sends the position.

This procedure is familiar to me, since I was an $\mathrm{HF} / \mathrm{DF}$ operator with the Royal Air Force in Malaya just after the Second World War.

\section{Errata}

(1) In the paper 'The Avoidance of Collisions for Newtonian Bodies with Hidden Variables', by B. D. Bramson (vol. 45, p. 55), equation (4.4) should read:

$$
t \leqslant 0 \vee t>0
$$

(2) In the paper 'Quantitative Estimation of Secondary Surveillance Radar Information, by D. J. A. Weeda, L. P. Ligthart, L. R. Nieuwkerk and B. C. M. van der Klein (vol. 45, pp. 26-35), the first initial of the last-named author was given as D instead of B.

On p. 27 line 22 , the phrase 'scans of 32 plots' should be replaced by 'scans of 32 numbers of plots'.

On p. 34, the denominator on the right-hand side of equation (1 2 ) should be $v(v \pm \Delta v)$ instead of $v(v \Delta v)$.

On p. 35 line 16 , 'air traffic route I' should be replaced by 'air traffic route $i$ '. 